\title{
En finir avec le déni des conséquences des accidents impossibles
}

\section{To finish with the denial of the consequences of impossible accidents}

\author{
Claude Pichot ${ }^{1}$ \\ ${ }^{1}$ Association française des ingénieurs et responsables de maintenance, France, claude.pichot@afim.asso.fr
}

RÉSUMÉ. Le déni de la possibilité des accidents dans le domaine industriel et de leurs conséquences catastrophiques reste le paradigme de notre modèle économique. II résulte de la vision à très court terme de l'économie qui vit les yeux rivés sur les cours de la Bourse alors que les installations industrielles vivent et se dégradent au rythme découlant des lois de la physique sur lesquelles sont construites les technologies. II existe une opposition théorique entre le profit à court terme et l'indispensable prise en compte du long terme pour éviter les accidents et les catastrophes.

Jusqu'à quand supporterons-nous en tant que citoyens que la fatalité serve d'excuse à l'examen sans complaisance des conséquences économiques et sociales des choix technologiques et des produits qui en découlent ? Nous ne sauverons notre planète que dans la mesure où nous aurons substitué le dogme de l'impossibilité des accidents à la prise en compte systématique de leur possibilité.

ABSTRACT. The paradigm of most of today's industrial business models seems to be the utter denial of the possibility of accidents occurring in industry and of their catastrophic consequences. The very short-term business vision of these models, concerned only with an economy which relies on stock prices, does not correlate with the reality of industrial plants which degrade before our very eyes according to the laws of physics. There is a distinct theoretical incompatibility between our current drive for short-term profit and the need for long-term solutions to avoid these kinds of accidents and disasters. How long will we continue to make excuses for the economic and social consequences that our technological choices and their resulting products have on us? We would save our planet if only we were able to replace this dogma of denial with a greater consideration for the mitigation of industrial accidents.

MOTS-CLÉS. accident, risque, maintenance.

KEY-WORDS. accident, risk, maintenance.

\section{Introduction}

Qu'elles soient subies, comme les inondations, les séismes, les éruptions volcaniques, les raz de marée, les tornades, etc. ou qu'elles soient engendrées par ses activités, l'histoire de l'Homme est jalonnée de catastrophes. La plombémie au temps de la Rome antique, les catastrophes minières des $\mathrm{XIX}^{\mathrm{e}}$ et $\mathrm{XX}^{\mathrm{e}}$ siècles, l'hydrargyrisme de la baie de Minamata, la destruction partielle de Fréjus avec la rupture du barrage de Malpasset, l'explosion de l'usine d'insecticides Bohpal en Inde, les effets de la dioxine à Seveso, l'explosion du réacteur de Tchernobyl, l'explosion d'AZF à Toulouse, l'incendie de la plateforme Deepwater Horizon, la fusion des réacteurs de Fukushima ou les accidents réputés impossibles comme la chute d'un générateur de vapeur ou l'incendie d'un condenseur à la centrale de Paluel, en font partie.

$\mathrm{Au}$ temps de Rome, après un séisme, une inondation, une éruption volcanique destructeurs et mortifères, le Sénat édictait des interdits de construire et d'habiter. Mais les hommes se réinstallaient sur les mêmes emplacements quelques décennies plus tard. La qualité des terres prévalait sur les dangers et les risques étaient vite oubliés. Les catastrophes humaines et environnementales n'ont pas manqué depuis la révolution industrielle. Comme elles ne manquent pas en ce début de XXI ${ }^{\mathrm{e}}$ siècle.

Depuis le début de la révolution industrielle, les dangers pour l'homme et l'environnement et les risques liés aux activités industrielles ont été considérés comme des conséquences «normales》du progrès industriel. Et la survenance des catastrophes a été souvent considérée comme relevant de la 
fatalité. C'est toujours après un accident que les règles de sécurité sont revues, que les évaluations des conséquences sont réalisées. Et la cause est rarement clairement identifiée, tout comme les insuffisances des méthodes utilisées pour la prévision des risques.

L'analyse des risques et la sûreté de fonctionnement qui se sont développées au cours du $20^{\text {ème }}$ siècle auraient dû apporter une réduction des risques, des accidents et de leurs conséquences humaines et environnementales. Mais les accidents avérés contredisent ce point de vue. Les postulats de l'analyse des risques et de la sûreté de fonctionnement occultent l'évolution dans le temps des risques liés au déficit du maintien en état de référence des matériels et des équipements au cours de leur durée de vie. Et c'est toujours après un accident que les impasses faites en conception se révèlent. L'accident survenu était «impossible» ou "n'aurait jamais dû se produire». C'est parce que l'accident est catégorisé impossible avec la conception retenue que les conséquences de sa survenance sur les personnes, l'environnement et les biens ne sont jamais étudiées et évaluées.

C'est 34 ans après le démarrage de Fessenheim que pour la première fois les conséquences économiques de la perte simultanée des sources électriques et de la source froide d'un réacteur nucléaire sont évaluées. Deux événements réputés impossibles qui ont cependant provoqué la catastrophe de Fukushima au Japon et la destruction de trois réacteurs en mars 2011.

Mais c'est aussi cinq ans après la réévaluation du niveau de sûreté des centrales $\operatorname{EDF}^{1}$ à la lumière du retour d'expérience de Fukushima que sont survenus deux accidents « impossibles ». L'incendie d'un condenseur en maintenance et la chute d'un générateur de vapeur en cours de manutention.

Le paradigme économique utilisé dans l'industrie nie les conséquences des accidents à très faible probabilité. Classés hors dimensionnement, ces accidents réputés impossibles ne font jamais l'objet d'une identification et de l'évaluation de leurs conséquences. Pas plus que ne sont étudiées les dispositions constructives et la mise en place des parades qui permettraient de limiter les conséquences sur les biens sans atteintes graves aux personnes et à l'environnement.

Mais les principes sur lesquels se fondent les études de sûreté de fonctionnement révèlent aussi d'autres lacunes. Dès lors que des prévisions de durée résiduelle de fonctionnement sans accident sont calculées sans que le déficit de maintien en état des biens soit mesuré, les conséquences de la survenance d'un accident avant la fin de la période sûre ne sont jamais prises en compte et évaluées. Le déraillement du Paris-Limoges et la catastrophe de Brétigny-sur-Orge en 2013 comme en 2016 l'accident d'hélicoptère Airbus en Norvège en témoignent.

Les accidents et leurs conséquences ne pourront être réduits que si ces dogmes sont remis en cause. Et que l'énergie susceptible d'être libérée en cas d'exposition à chaque danger identifié quelle qu'en soit l'occurrence ne porte pas atteinte à la santé des personnes, à l'intégrité des biens et à l'environnement. Mais la prise en compte du coût des conséquences des événements réputés impossibles aurait conduit à ne pas réaliser certaines installations.

Notre réflexion s'articulera autour des points suivants: 1/ nous montrerons que l'approche probabiliste, qui fait office ce paradigme depuis de longues années, ne met pas à l'abri des catastrophes, 2/ mais que les dogmes ont la vie dure ; 3/ malgré une catastrophe récente de grande ampleur comme celle d'AZF à Toulouse en septembre 2001 ; 4/ pourtant, si la maintenance est censée coûter cher, elle s'avère peu coûteuse au regard du montant des dépenses nécessaires pour faire face à l'après-catastrophe, $5 /$ comme le démontrent au centuple de nombreux exemples. Nous conclurons nos propos par quelques considérations d'ordre général sur les conséquences des activités humaines sur la nature qu'elles contribuent inexorablement à dégrader. 


\section{L'approche probabiliste des risques ne met pas à l'abri des catastrophes}

Les accidents nucléaires survenus sur le site de Fukushima après le tsunami du 11 mars 2011 posent encore une fois la question des postulats qui organisent la défense en profondeur des installations industrielles. En ayant considéré que la perte de la source froide et des sources électriques avait une probabilité de survenance très faible dans une région qui subit $20 \%$ des séismes et des tsunamis de la planète, rien n'a été prévu pour y faire face. Il n'a pas été imaginé qu'un tsunami puisse engendrer une vague de plus de 10 mètres dans une région qu'une vague de plus de 20 mètres avait submergée en 1923. Mais il n'a pas été pris en compte non plus que la submersion des diesels électriques de secours pouvait aussi entraîner leur ruine mécanique, l'eau étant incompressible. Ce qui s'est passé au Japon fait ressortir l'état criant des déficits de prise en compte des conséquences d'un accident réputé ne jamais pouvoir survenir. Car si l'accident de Fukushima est devenu une catastrophe, c'est parce que de l'énergie électrique et de l'eau traitée n'auront pas été apportées dans des délais compatibles avec le rétablissement d'un état sûr et cela parce que personne n'avait imaginé qu'il faudrait y faire face un jour.

Autrement dit, la compagnie japonaise d'électricité TEPCO aura perdu plusieurs dizaines de milliards d'euros d'actifs, stérilisé définitivement le site, déplacé plusieurs centaines de milliers de personnes et contaminé combien d'entre elles pour ne pas avoir dépensé quelques centaines de millions d'euros dans la mise au point des alternatives à la perte simultanée des sources froide et électrique. Plus de cinq ans après, la situation de sûreté des réacteurs n'est toujours pas stabilisée. Et le coût de la catastrophe dépasse les 80 milliards d'euros.

Parce qu'ils ont la charge de maintenir les équipements, les responsables de maintenance sont particulièrement concernés par les accidents, car ils sont les premiers intervenants avec leurs équipes dans ces situations de crise. Ils sont aussi ceux qui connaissent le mieux l'état des équipements et les déficits techniques découlant de budgets insuffisants alloués à la maintenance par les actionnaires. Ils sont confrontés aux conséquences des hypothèses retenues lors de la conception concernant la disponibilité des moyens ultimes de défense en cas d'accident. Enfin, ils sont ceux qui dirigent des intervenants de grande technicité les plus exposés aux accidents et maladies professionnelles.

En France, malgré la catastrophe de Tchernobyl en 1986, il aura fallu attendre celle de Fukushima pour que l'IRSN (Institut de radioprotection et de sûreté nucléaire) procède en 2011, 34 ans après le démarrage des réacteurs de la centrale de Fessenheim à l'évaluation du coût d'un accident majeur survenant sur un site nucléaire de production d'électricité. Ce qui a fait de la perte simultanée de la source froide et des sources électriques un événement avéré, alors qu'il avait été considéré jusqu'alors comme « impossible ».

Ainsi, pour le site de Fessenheim, la perte totale de la source froide du fait d'une brèche du Grand canal d'Alsace entrainant aussi la submersion de toutes les sources électriques normales et secourues du site n'est plus un « évènement impossible ».

EDF convenait, le 31 mars 20112, en réponse à l'ASN (Autorité de sûreté nucléaire) que l'organisation nationale de crise en vigueur, bien que très expérimentée et entraînée, ne disposait pas au niveau national des moyens matériels permettant d'apporter rapidement (dans les 24 à 48 heures) une aide matérielle à un site ayant perdu toute source d'alimentation électrique ainsi que toute source de refroidissement et d'appoint en eau. De même que les moyens humains et matériels sur site ne permettaient pas d'éviter qu'une perte totale et cumulée des sources électriques et de refroidissement ne conduise à un accident majeur avec dégradation du combustible en piscine de désactivation ou du cœur et du confinement du réacteur. 
Selon les études effectuées en 2013 par l'IRSN ${ }^{3}$ (Institut de radioprotection et de sûreté nucléaire), le coût d'un accident grave correspondant à la fusion du cœur d'un réacteur de $900 \mathrm{MW}$ avec des rejets contrôlés et filtrés dans l'environnement est évalué à 120 milliards d'euros soit $6 \%$ du PIB. Dans le cas d'un accident majeur avec des rejets massifs et non contrôlés dans l'environnement, le coût pourrait atteindre 430 milliards d'euros, soit plus de $20 \%$ du PIB. A ces coûts économiques, il faut rajouter les conséquences radiologiques pour les populations et les effets à long terme sur la santé.

Si ces évaluations avaient été réalisées en 1971, avant le lancement de la filière électronucléaire des réacteurs pressurisés à eau légère, les alternatives de production de l'électricité à partir d'énergies renouvelables auraient pu être étudiées.

Mais le dogme qui consiste encore à considérer qu'une probabilité très faible de survenance d'un évènement catastrophique nous délivrerait d'étudier les conséquences de l'accident et de mettre en place les parades qui éviteraient que l'accident ne tourne à la catastrophe a la vie dure malgré des accidents avérés. Et certaines hypothèses ont la vie très dure !

Ainsi l'approche probabiliste qui sert toujours de base à la détermination de l'occurrence d'accident grave n'a pas varié depuis 40 ans (1/100000 par année-réacteur).

Pourtant, les accidents majeurs de Tchernobyl et de Fukushima et l'accident grave de Three Mile Island aux Etats-Unis, nous invitent à remettre en cause cette probabilité, car il s'agit d'accidents avérés.

Le 5 mai 2011 Jacques Repussard, directeur général de l'IRSN, dans une interview parue dans $L e$ Monde relève que «sur le parc mondial, 14000 années-réacteur sont déjà passées, et les statistiques montrent qu'on est à $0,0002(2.10-4)$ accident nucléaire grave par an, soit vingt fois plus qu'attendu selon les études probabilistes, qui ne savent pas bien prendre en compte l'aléa naturel et le facteur humain. Le nucléaire fait jeu égal avec l'industrie chimique. C'est insuffisant. On peut donc se poser la question : l'homme est-il en mesure de maîtriser cette technologie pour diviser au moins par deux ce risque d'accident? Y a-t-il une barrière ? Ce serait une conclusion inquiétante, car cela signifierait qu'avec 1000 réacteurs installés, un accident nucléaire grave se produirait en moyenne tous les dix ans, ce qui n'est pas supportable».

Ainsi, malgré des accidents avérés qui se révèlent au moins 20 fois plus fréquents que prévu, le changement de paradigme n'est pas à l'ordre du jour.

Les conséquences catastrophiques de l'activité industrielle ne doivent pas être pointées du doigt. Car pour la plupart des investisseurs, «s'il fallait prendre en compte les conséquences des accidents à la conception, on ne ferait plus rien ».

Sur la base du coût initial de l'EPR de Flamanville, le coût d'un accident majeur (430 milliards d'euros) équivaut au coût de construction de 122 réacteurs. Avec un coût réévalué de construction par réacteur de 8,5 milliards d'euros, le même accident majeur équivaut à la construction de plus de 50 réacteurs. Et cela alors que le parc nucléaire français compte 58 réacteurs en service aujourd'hui ! Procéder à cette évaluation aurait dû conduire à rechercher des alternatives et à proposer d'autres voies de production d'une électricité non nucléaire et non carbonée.

Après Fukushima, il faut oser dire qu'un accident majeur, certes rarissime, est inéluctable et qu'il faut changer de paradigme. Ce changement de paradigme ne contredit pas les principes de sûreté industrielle et nucléaire. Il conduit à envisager des modes additionnels de gestion des risques et de leur pédagogie. Car la prévention des risques ne suffit pas. Elle doit être accompagnée des moyens de faire face à l'accident et à ses conséquences humaines, sanitaires et économiques.

3. http://www.irsn.fr/FR/connaissances/Installations_nucleaires/Les-accidents-nucleaires/cout-economique-accident/Pages/2-couteconomique-pour-2-scenarios.aspx? $d I d=b 5 e 6 d 248-170 f-4 f 79-a 514-8 f b 9 c b 86 a 6 d 5 \& d w I d=0 d d 84144-5 e 15-4 e 92-b 9 e f-b e 117439 b 36 b$ (c) 2016 ISTE OpenScience - Published by ISTE Ltd. London, UK - openscience.fr Page $\mid 4$ 
Sans la remise en cause de cette infaillibilité doctrinale, nous ne sommes pas à l'abri d'une catastrophe analogue à celle de Fukushima. Car à considérer toujours qu'une probabilité de survenance d'accident est très faible, l'étude de ses conséquences et de la mise en place des parades n'est jamais faite pour éviter que l'accident ne finisse en catastrophe.

Quels dogmes faut-il remettre en cause ? Le premier est celui de la perte des sources électriques consécutives à la submersion des installations. Admettre la submersion c'est admettre la perte des alimentations électriques normales et de secours si elles ne sont pas protégées. Qu'en aurait-il coûté de l'avoir étudié au préalable? Des portes étanches dans les parkings protègent bien nos véhicules lors des crues de la Seine, mais rien d'équivalent pour les groupes diesels de secours des centrales, ni pour les systèmes de sauvegarde essentiels et leurs alimentations électriques.

Pour les centrales en bord de falaise, le positionnement des sources électriques de secours et d'ultime secours en haut des falaises rendrait le système plus sûr à la conception. Mais où se trouvent donc ces groupes dans nos centrales de bord de mer?

Le second dogme est celui de l'impossibilité de perte de la source froide. Admettre sa perte, c'est s'imposer d'étudier comment disposer d'une source froide de substitution quand la prise d'eau brute est tarie ou accidentée et que l'énergie électrique fait défaut. Seuls les sites de Civaux et de Cattenom disposent d'une source froide de réserve de plusieurs semaines.

Qu'aurait coûté la réalisation d'une barge de secours capable de fournir de l'énergie électrique, de disposer d'une source froide auxiliaire, d'eau borée, d'une centrale de ventilation de secours, etc. ?

Qu'auraient coûté des pompes mécaniques de secours ? Face à la perte des sources électriques et de la source froide, la fusion du combustible est inévitable. Avec pour conséquences des débits de rayonnements dangereux ou mortels pour les exploitants et des contaminations graves pour l'environnement. Mais qu'aurait coûté de prévoir à la conception des accès souterrains permettant le repli et la relève des exploitants depuis les salles de commande vers des lieux protégés ?

Et si la fusion du combustible associée à celle des aciers du réacteur avait été admise, qu'aurait coûté la conception d'un cendrier capable de contenir et de refroidir sans risque la masse en fusion sans disperser de contamination dans l'eau ou dans l'air?

Il est plus que temps de revoir l'approche probabiliste des risques car elle ne nous met pas à l'abri des catastrophes. Mais les dogmes ont la vie dure, très très dure !

\section{Les dogmes ont la vie dure...}

Après l'accident de Fukushima, EDF a repris les analyses de sûreté et pris en compte le nouveau paradigme de la perte simultanée du refroidissement et des sources électriques. Ce qui a conduit à des mesures techniques et organisationnelles de mise à niveau « post Fukushima » de plus de 10 milliards d'euros dont la mise en œuvre s'étalera jusqu'au-delà de 2020.

Mais cette réévaluation de la sûreté n'a pas pourtant fait varier EDF sur le dogme de l'accident impossible. Car, le 31 mars 2016, sur le site de Paluel, dans le cadre des opérations de maintenance « de grand carénage » du réacteur $\mathrm{n}^{\circ} 2$ à l'arrêt, la chute d'un générateur de vapeur usagé ${ }^{4}$ lors de sa manutention d'extraction du bâtiment réacteur a balayé cette hypothèse de la chute "impossible ». Hypothèse pourtant contestée par l'IRSN depuis la conception des réacteurs et confirmée par Jacques Repussard, le directeur de l'IRSN après l'accident de Fukushima qui estime que cet incident inédit n'a pas été suffisamment anticipé ${ }^{5}$. 
«Lors de la conception des réacteurs, dans les années 1990, l'IRSN a souhaité que le scénario de la chute d'un générateur de vapeur soit explicitement pris en compte afin de démontrer que cela n'aurait pas de conséquences sur le bâtiment »... « Mais EDF n'a pas donné suite, considérant qu'un tel scénario était impossible, les matériels de manutention étant justement conçus pour qu'un tel événement n'arrive jamais ».

La rupture du système d'assemblage du palonnier de manutention du générateur de vapeur sur le câble de levage a entraîné la chute des 465 tonnes du générateur de vapeur sur le plancher de la piscine vide du réacteur revêtu de ses plateaux de protection. Elle a aussi balayé le scénario impossible...

L'énergie libérée par cette chute a été encaissée par les ouvrages de génie civil de la piscine et du réacteur. Mais du fait de «l'impossibilité » de la chute, c'est seulement lorsque les calculs pour évaluer les dommages effectifs seront disponibles, que l'on saura si les ouvrages et les équipements affectés par la chute ont conservé leur capacité de résister aux efforts résultants des hypothèses de calcul initiales. Ou si les désordres consécutifs à cette chute « impossible » remettent en cause la sûreté du bâtiment.

Plus de 6 mois après l'accident, l'absence d'information publique émanant d'EDF et de l'ASN laisse augurer, contrairement à ce qu'a toujours affirmé EDF que la chute d'un générateur de vapeur a entrainé des contraintes qui ont dépassé les contraintes maximales liées au séisme.

Tant que le changement de paradigme sur «l'impossibilité » ne sera pas remis en cause pour les phases de maintenance comme cela est maintenant admis pour les phases de production, des accidents continueront de survenir. Et la fatalité toujours invoquée.

Cette attitude qui consiste à évacuer les conséquences lointaines des aléas de l'exploitation des patrimoines est la conséquence d'un modèle économique orienté sur la rentabilité à court terme. Et peu enclin à prendre en compte les conséquences à moyen et long terme de l'exploitation sur de très longues périodes de vie des patrimoines industriels.

\section{L'après-AZF}

Trente et une victimes, des milliers de blessés, des dizaines de milliers d'habitants privés de fenêtres, des dégâts matériels chiffrés en milliards d'euros : ce qui fait de l'accident survenu le 21 septembre 2001 dans l'usine AZF de Toulouse une tragédie, ce sont ses conséquences insupportables.

Seule l'absolue certitude que le risque était trop faible a pu conduire AZF à stocker plusieurs centaines de tonnes d'une matière potentiellement explosive sans en évaluer les conséquences alors que le site était certifié ISO 14001 pour le management environnemental et que malgré ce cadre, le nitrate d'ammonium n'avait pas été identifié comme potentiellement explosible. Et pourtant selon la base de données ARIA ${ }^{6}$, depuis 1975, 20 accidents graves mettant en cause le nitrate d'ammonium étaient survenus dans le monde avant celui d'AZF. Soit un accident tous les 9 mois.

Pourtant, c'est bien parce que le risque zéro n'existe pas, parce que l'incertitude demeure, qu'il faut examiner tous les effets possibles d'un accident - quel qu'il soit - afin de concevoir le dispositif permettant d'éviter qu'il ne tourne à la catastrophe.

Par-delà les morts, les blessés et le traumatisme causé dans la population, le drame de Toulouse invite à quitter le terrain de la certitude et à examiner si les surcoûts nécessaires à une sécurité en profondeur ne sont pas les plus compatibles avec la rationalité économique. Le seul préjudice financier pour les citoyens et les actionnaires est à ce jour supérieur à 2,5 milliards d'euros ${ }^{7}$. Les actionnaires ont

\footnotetext{
${ }^{6}$ Base de données publique ARIA www.aria.developpement-durable.gouv.fr/resultat-recherche-accident/

${ }^{7}$ Soit plus de 50 ans du résultat net du site d'AZF en 2001

(c) 2016 ISTE OpenScience - Published by ISTE Ltd. London, UK - openscience.fr 
dû procéder à une augmentation de capital de 500 millions d'euros pour faire face aux coûts non pris en charge par les assureurs. Et ce alors que la situation nette était de 135 millions d'euros au 30 juin 2001.

D'un point de vue froidement financier, qu'aurait coûté l'évaluation de l'impact d'une explosion « improbable » : un million d'euros ? Au vu de l'ampleur des conséquences calculées, la raison aurait certainement repris ses droits. Et il n'aurait sûrement pas fallu dépenser 50 millions d'euros dans la sécurisation des installations pour faire en sorte que l'événement du 21 septembre 2001 reste un accident et ne devienne jamais une tragédie.

Cet événement aurait dû changer radicalement notre comportement face aux risques. Il ne devrait plus être admissible de concevoir, construire et exploiter des usines sans avoir procédé à un inventaire exhaustif de tous les risques et examiné toutes leurs conséquences. Et cela lors de toutes les phases de la vie des installations (construction, exploitation, maintenance, déconstruction). Alors qu'aujourd'hui encore, au motif que la probabilité d'occurrence de tel ou tel risque est jugée trop faible, l'examen de ses conséquences n'est pas réalisé. Un accident qui a très peu de chances de se produire ne saurait être décrété hors dimensionnement dès lors que ses conséquences potentielles sont catastrophiques.

Depuis l'accident d'AZF en 2001, le recensement international des accidents de la base ARIA dénombre 10 événements mettant en cause le nitrate d'ammonium, soit un accident tous les 8 mois. Malgré les discours sur l'amélioration de la sécurité de ces installations, l'occurrence d'accidents avérés a augmenté depuis l'accident d'AZF. Ce qui ne plaide pas en faveur d'un changement d'attitude des industriels face aux risques liés au nitrate d'ammonium.

Il faut aussi s'interroger sur le fait que la Cour de cassation ait ouvert le 13 janvier 2015 la voie à un nouveau procès pour juger des responsabilités dans l'explosion du complexe chimique AZF en cassant l'arrêt de la cour d'appel de Toulouse, plus de treize ans après la catastrophe qui avait coûté la vie à 31 personnes et fait 2.500 blessés et détruit les sites de production de Grande Paroisse.

En septembre 2012, la cour d'appel de Toulouse avait reconnu la responsabilité de l'ex-directeur du complexe chimique et de la société Grande Paroisse, propriétaire du site et filiale de Total. Grande Paroisse avait été condamnée à 225000 euros d'amende, la peine maximale, et l'ex-directeur à trois ans de prison, dont deux ans avec sursis et à 45000 euros d'amende. La cour d'appel avait cependant écarté la responsabilité du groupe Total, propriétaire de Grande Paroisse, et de son ancien PDG Thierry Desmarest.

La Cour de cassation, qui a renvoyé l'affaire en appel à Paris, a retenu notamment l'argument concernant l'impartialité de l'une des magistrates de Toulouse. Ainsi, près de 15 ans après les faits, personne ne sait vraiment ce qui s'est passé le 21 septembre 2001. Le modèle économique qui gouverne repousse toujours à plus tard la reconnaissance des faits et des erreurs commises. Ces calculs et les arguties juridiques qui les accompagnent font ainsi peu de cas des victimes et des blessés.

La loi sur les risques technologiques ${ }^{8}$ votée en juillet 2013 après le rapport de la commission d'enquête parlementaire ${ }^{9}$ sur la catastrophe d'AZF a défini l'obligation des études de danger, ce qui constituait une avancée significative en matière de prise en compte des risques technologiques. La loi a aussi précisé les modalités de diffusion des informations au travers des commissions locales d'information. Mais une subtilité juridique modifiant un article du code du commerce permet aux exploitants de s'exonérer des conséquences des études de danger.

Ainsi la loi no 2003-699 du 30 juillet 2003 relative à la prévention des risques technologiques et naturels et à la réparation des dommages prévoit que «tout exploitant d'un établissement comportant au moins une installation soumise à servitude d'utilité publique ou visée à l'article 3-1 du code minier

8. https://www.legifrance.gouv.fr/affichTexte.do?cidTexte=JORFTEXT000000604335\&categorieLien=id

9. http://www.assemblee-nationale.fr/rap-enq/r3559/r3559-011.asp\#annexe1

(C) 2016 ISTE OpenScience - Published by ISTE Ltd. London, UK - openscience.fr 
procède à une estimation de la probabilité d'occurrence et du coût des dommages matériels potentiels aux tiers, en cas d'accident majeur survenant dans cette installation et pouvant présenter des effets graves sur les biens situés à l'extérieur de l'établissement ${ }^{10}{ }^{1}$.

Il est précisé dans cette loi que l'estimation :

- « est réalisée pour chacun des accidents majeurs identifiés dans l'étude des dangers »;

- « est révisée à l'occasion des révisions de l'étude des dangers précitée »;

- « n'est pas opposable à l'exploitant par les tiers en cas de litige lié à un accident survenant dans l'installation ».

Ne sont pas opposables à l'exploitant, c'est-à-dire, ne peuvent pas être prises en compte par le juge pour des litiges résultant de l'accident invoqué par des tiers. Ainsi l'estimation du coût des dommages matériels potentiels aux tiers exigée de l'exploitant ne peut en aucun cas servir de base au jugement. Ce qui montre encore la difficulté du modèle économique à se préoccuper équitablement des conséquences des accidents.

\section{Essayez les pannes, ou les accidents si vous trouvez que la maintenance coûte cher...}

\subsection{La maintenance coûte cher entend-on souvent}

En France les dépenses annuelles de maintenance dépassent les 64 milliards d'euros, soit $3 \%$ du $\mathrm{PIB}^{11}$ :

- Secteur industriel et transport : 21,5 milliards d'euros ;

- Secteur immobilier et tertiaire : 17,6 milliards d'euros ;

- Réparation des véhicules : 20 milliards d'euros ;

- Secteur Défense : 3,1 milliards d'euros ;

- Transport aérien : 2 milliards d'euros;

-Réparation navale : 0,3 milliard d'euros.

Contraindre les budgets de maintenance est le plus mauvais calcul en termes de sécurité, de service rendu et de profit durable. Combien faudra-t-il encore d'accidents graves et de morts, combien encore de pannes de réseaux de communication pour que les décideurs le comprennent?

Les pannes ne découlent pas des tableaux de bord des gestionnaires, mais de la fatigue des matériels résultant de leur degré de sollicitation. Les conséquences des pannes: des anomalies de fonctionnement, des échauffements, des ruptures de composants... Ce qui, a minima, entraine la perte du résultat attendu mais conduit aussi à des accidents de personnes, à des atteintes à l'environnement et à des catastrophes.

Les pannes, comme les accidents sont des événements rarement liés à la force majeure. Ils découlent du déficit de prise en compte des effets des énergies pouvant être libérées en cas de dysfonctionnement. Et de programmes de maintenance ne couvrant pas tous les modes de défaillance prévisibles des matériels et des systèmes. Le déficit de maintenance prend sa racine dans les défauts de surveillance des composants critiques, des zones chargées ou des capteurs de sécurité... Et aucun patrimoine n'est épargné. 
La nature a vite fait de reprendre ses droits sur un ouvrage sans surveillance. Les efforts engendrés par la croissance de végétaux non désirés conduisent des bâtiments, des équipements ou des ouvrages à la ruine.

De même que les défauts d'étanchéité des toitures amènent des infiltrations dans les structures en béton armé des bâtiments. Sans être éliminées, ces infiltrations conduisent en quelques années à des éclatements du béton d'enrobage des armatures en acier et à des désordres au pied des ouvrages. Tout simplement du fait de la pression exercée par la colonne d'eau infiltrée. Ah si Pascal savait que les résultats sa célèbre expérience du crève-tonneau n'a pas vraiment incité, plus de 350 ans après sa mort, les investisseurs à s'en préoccuper.

De même que l'élévation de la température ambiante provoque des dilatations dans les rails de chemin de fer. Lesquelles, combinées aux efforts de fatigue dus au passage des trains peuvent conduire à la rupture des composants.

Garder en état les matériels, les équipements, les infrastructures, les ouvrages, c'est se préoccuper de l'effet des énergies auxquelles ils sont soumis ou risquent de l'être. Se préoccuper des pannes, c'est analyser les conséquences des énergies présentes ou potentielles sur les équipements. Lesquelles découlent de la fréquence des sollicitations, de leur intensité et de leur durée. Le modèle économique actuel donne la priorité absolue au cours terme. La consolidation trimestrielle des résultats des entreprises ne fait pas bon ménage avec la prise en compte de l'usure et des dégradations consécutives à l'usage des équipements, des machines, des patrimoines privés ou publics au cours du temps. Il n'est pas raisonnable de piloter la maintenance les yeux rivés sur l'indice du CAC 40.

Depuis quand les éclisses de l'aiguillage rompues le 12 juillet 2013 au passage du train Intercités $\mathrm{n}^{\circ} 3657$ à Brétigny-sur-Orge étaient installées et quand, comment et à quelle périodicité leur santé a été contrôlée ? Les proches des sept victimes, les 32 blessés dont 11 grièvement et plus de 200 personnes extrêmement choquées ou ayant subi des préjudices matériels ont le droit de savoir.

Seule la réponse à ces questions permettra d'améliorer la sécurité des trains au passage des aiguillages. Les conclusions de rapport d'enquête du BEATT ${ }^{12}$ fournissent des éléments de réponse et pointent le déficit de maintenance des aiguillages. Et ce déficit ne datait pas de la veille.

Les enquêteurs relèvent que des défauts sur le cœur de l'aiguillage incriminé étaient identifiés depuis plus de cinq ans avant l'accident. Mais le remplacement de ce composant était planifié pour 2015.

Sans doute, on ne saura jamais qui a réalisé les études de sûreté de fonctionnement et qui a pris la décision de différer ce remplacement.

Le BEATT ${ }^{13}$ relève «le peu de rigueur qui a dû présider à la vérification dont cette traversée de jonction double a fait l'objet en novembre 2011 pour que la fissuration qui s'était développée dans l'âme de l'about de cœur n'ait pas été signalée alors qu'elle devait vraisemblablement être très avancée ».

Il est cependant des défaillances qui introduisent seulement des pertes économiques et des perturbations pour les usagers. C'est le cas de la panne du réseau SFR qui a privé, en mars 2015, 3,8 millions abonnés de SFR de communications pendant près de 5 heures. En cause, un HLR (home location register), un équipement stratégique pour localiser et connecter les abonnés. Le dogme de «l'impossibilité » revient. C'est au matériel qu'incombe la faute : «le HLR, un équipement qui

12. BEATT : bureau d'enquête accidents dans les transports, rapport $n^{\circ}$ BEATT 2013-009 d'enquête sur le déraillement du train Intercités $n^{\circ} 3057$ le 12 juillet 2013 à Brétigny-sur-Orge.

13. Rapport BEATT 2013-009, page 16.

(c) 2016 ISTE OpenScience - Published by ISTE Ltd. London, UK - openscience.fr

Page $\mid 9$ 
n'aurait jamais dû tomber en panne » selon SFR. Mais dont un jumeau avait été à l'origine d'une panne massive de 11 heures sur le réseau d'Orange en 2012 !

\subsection{La prise en compte de l'expérience reste à améliorer}

N'en déplaise aux tenants de la primauté du modèle économique sur le modèle technologique, les matériels défaillent selon les lois de la physique. Lois qui n'ont rien à voir avec les lois du moment que sont la loi du marché, la mondialisation des échanges commerciaux, etc.

Les matériels se moquent des dogmes, ils cassent selon les lois de la mécanique quand les contraintes auxquelles ils sont soumis dépassent les limites de résistance. Ou ils s'échauffent selon les lois de la thermique et quand les échauffements sont trop élevés, les informations erronées ou l'incendie ne sont jamais loin.

La maîtrise de la technologie est au cœur de l'efficacité. Si « science sans conscience n'est que ruine de l'âme », les patrimoines sans maintenance ne sont que des ruines en devenir. Il est grand temps de développer les méthodes de prévention des dysfonctionnements, ce qui nécessite d'investir dans la matière grise et la formation technologique. Donc de dépenser plus, pour fonctionner plus et gagner plus.

\section{La vanne qui valait 20 milliards de dollars, un bras de $\mathbf{4 0}$ en plus !}

Dans la Lettre de la Maintenance de l'AFIM de juin 2010, j'avais écrit :

«19 avril 2010, incendie suivi d'une explosion sur la plateforme Deepwater Horizon à $80 \mathrm{~km}$ au large de la Floride. 11 morts et 17 blessés sur les 126 personnes présentes à bord de la plateforme. C'est le début d'une des plus grandes catastrophes écologiques de l'histoire des Etats-Unis. Deux jours après l'incendie, naufrage de la plateforme semi submersible avec une fuite de brut depuis la tête du puits située à plus de 1500 mètres de profondeur.

Dans les premiers jours, l'opérateur BP a affirmé maîtriser la situation. Sur Internet, grâce à la RVA (réalité virtuelle augmentée), un robot sans doute virtuel lui aussi, colmatait la fuite selon un séquençage sans faille. Mais la RMA (Réalité Matérielle Avérée) s'est obstinée à refuser le colmatage si bien réussi en RVA ! Cela fait plus de deux mois que plusieurs milliers de $\mathrm{m}^{3}$ de pétrole brut se déversent dans le golfe du Mexique car l'accident a empêché le fonctionnement de la vanne de sécurité. Les dégâts sont estimés à 20 milliards de dollars ».

Le 19 septembre, cinq mois après le début de l'accident, la fuite est maîtrisée après un déversement de plus de 630000 tonnes de pétrole dans le golfe du Mexique. Le coût est aujourd'hui évalué à 60 milliards de dollars. Et il a fallu boucher le puits avec un forage dévié.

«L'enquête sur l'explosion de la plateforme Deepwater Horizon, le 20 avril dernier, a révélé que l'installation souffrait de nombreux problèmes d'entretien. Stephen Bertone, ingénieur en chef de la compagnie suisse Transocean, a indiqué lundi devant la commission chargée de faire la lumière sur les causes de la catastrophe que la plate-forme de Transocean, qui forait le puits pour le compte de BP, devait faire l'objet d'opérations de maintenance «excessives» que son équipe n'a pu réaliser. Une liste des tâches à accomplir, dressée à l'issue d'un audit réalisé en 2009, en répertorie 390, qui auraient demandé 3.500 heures de travail, a précisé Richard Godfrey, avocat de BP. L'explosion de la plateforme, qui a fait onze morts, a déjà coûté 3,95 milliards de dollars à BP en travaux de 
colmatage et de nettoyage. La marée noire aurait libéré jusqu'à 630000 tonnes de brut dans le Golfe du Mexique, soit trois plus que lors du naufrage de l'Amoco Cadiz ${ }^{14} »$.

Ainsi un déficit de maintenance de 3500 heures, 2 à 3 millions de dollars, a engendré une catastrophe humaine, écologique et économique avec 11 morts et 17 blessés et 60 milliards de dollars.

Encore une fois, la double négation de l'impossibilité de la défaillance d'une vanne de sécurité et l'absence de dispositif redondant et l'impossibilité d'une explosion due à un déficit avéré de maintenance de la plateforme elle-même ont prévalu.

Quel investisseur aurait engagé des fonds dans ce forage profond si les évaluations des conséquences d'un accident avaient été connues? En l'espèce, l'investisseur BP a laissé faire en connaissance de cause. Il n'ignorait pas le déficit de maintenance de la plateforme Deeepwater Horizon, pas plus qu'il ignorait l'absence de redondance de la vanne d'isolement du forage.

Mais comme il n'est pas de frein moral dans le modèle économique actuel, l'espérance de profits hors normes fait sauter tous les garde-fous. Les poissons et la nature ne font jamais entendre leur voix.

\section{Conclusion : ce n'est pas l'homme qui réchauffe la nature mais l'inverse}

Le modèle économique dominant et la pensée qui l'accompagne sont solidement ancrés sur le caractère " impossible » des conséquences de l'activité humaine. A l'heure où la quantité de mercure, de polychlorobiphényle augmente de façon alarmante dans les poissons que nous mangeons, à l'heure où le niveau des mers ne cesse de monter, le camp des tenants du modèle économique dominant donne de nouveau de la voix.

Avec le Grenelle de l'environnement et les travaux du $\operatorname{GIEC}^{15}$ nous pouvions penser que le lien entre l'activité industrielle et le réchauffement climatique était maintenant admis par tous. Et que le cercle vertueux de la réduction des impacts négatifs de l'activité industrielle était enclenché. Mais voilà que l'ancien président de la République qui a lancé au cours de son mandat le Grenelle de l'environnement conteste que l'homme et les activités industrielles soient en quoi que ce soit impliquées dans les pollutions environnementales et climatiques de notre planète.

Devant l'Institut de l'entreprise, l'ancien chef de l'Etat a déclaré, selon des propos rapportés par son directeur de campagne, Gérald Darmanin ${ }^{16}$ :

«On a fait une conférence sur le climat. On parle beaucoup de dérèglement climatique, c'est très intéressant, mais ça fait 4,5 milliards d'années que le climat change. L'homme n'est pas le seul responsable de ce changement. Je préférerais qu'on parle d'un sujet plus important: le choc démographique. La France doit porter une conférence sur la démographie. Jamais la terre n'a connu un choc démographique tel qu'elle va le connaître, puisque nous serons onze milliards dans quelques années. Là, l'homme en est directement responsable. Et personne n'en parle. »

Pour le climatologue Jean Jouzel, ancien vice-président du GIEC, le Groupe d'experts intergouvernemental sur l'évolution du climat, dont les travaux font autorité, les déclarations de Nicolas Sarkozy sont "vraiment pitoyables pour quelqu'un qui a lancé le Grenelle de l'environnement » sous son quinquennat et traduisent un "mépris de la communauté scientifique. »

On peut s'interroger sur ce qui a inspiré Nicolas Sarkozy. Oser prétendre que les activités industrielles ne sont pas responsables des atteintes à la planète c'est accréditer l'idée que des produits

14. Maintenance insuffisante, Le Figaro, 20/07/2010.

15. Le GIEC : Groupe d'experts intergouvernemental sur l'évolution du climat.

16. Le Monde, 15/09/2016.

(c) 2016 ISTE OpenScience - Published by ISTE Ltd. London, UK - openscience.fr 
qui n'existent pas à l'état de nature auraient été en fait produits à l'insu de leur plein gré par la nature. C'est tout simplement absurde et mensonger. C'est une contre vérité scientifique qui éclaire l'âpreté du débat qui oppose les visions à très court terme à celles du moyen et long terme.

Les tenants de l'accroissement continu du rendement des capitaux mènent l'Homme à sa perte en exigeant toujours plus. Leur credo est toujours « agir sans contraintes car nous sommes le progrès ».

Qui se souvient que depuis le début de l'ère industrielle, il y a à peine 150 ans, nous avons épuisé en métropole les ressources minérales de notre sous-sol. Finis le minerai de fer et le charbon, épuisée la bauxite, disparus les phosphates, dévoré l'uranium... Et polluées les nappes phréatiques avec les nitrates de l'agriculture, les cours d'eau avec le tritium, les PCB, etc.

La liste des atteintes à notre environnement est longue et affirmer que l'homme n'y est pour rien relève de l'ignorance des sciences et des technologies, et du mépris pour l'intelligence des citoyens. Quand ce discours est tenu par un homme d'Etat, c'est la bêtise qu'on prépare à entrer au Panthéon.

Le débat sur la prise en compte des conséquences de l'activité industrielle dans les décisions d'investissements ne doit plus être occulté par la liberté d'entreprendre. La liberté d'entreprendre est indispensable, mais elle doit s'accompagner de la prise en compte des effets à moyen et long terme des conséquences des choix industriels.

Nous avons aujourd'hui accumulé suffisamment de connaissances pour que la projection des conséquences de nos décisions dans le futur fasse partie de notre mode de fonctionnement en société. Nous ne pourrons plus longtemps invoquer la fatalité en regard des accidents car les citoyens ne l'admettent plus.

Comme ils admettent de moins en moins facilement de devoir supporter seuls les conséquences des catastrophes. Alors que les propriétaires actionnaires des compagnies industrielles ne sont que très rarement ruinés par ces catastrophes. Il était inimaginable qu'un des pays les plus avertis en matière technologique comme le Japon connaisse une catastrophe nucléaire. Mais c'est arrivé et des événements «complètement inimaginables» ont surgi de «l'impossible». Et ils continueront de le faire si nous ne changeons pas de paradigme. Car la logique de la démesure, que les Grecs appelaient l'«hybris ", l'orgueil qui nous pousse à dépasser la mesure et à ne pas vouloir évaluer les conséquences de notre démesure guide notre modèle économique. Cette idée résonne fortement dans notre culture car nous faisons preuve d'une avidité sans fin alors même que notre Terre atteint sa limite de l'absorption sans dommage des effets de l'activité humaine.

L'ampleur de la consommation des matières premières non renouvelables, des émissions toujours croissantes des gaz à effet de serre, de la disparition des espèces, de la pollution des sols et de l'air et de l'eau témoignent de notre avidité. Laquelle se nourrit de notre croyance que la technologie pourra compenser ou éviter le dommage. Jusqu'à ce que l'accident survienne, entraînant des conséquences bien pires que celles qu'on pouvait imaginer. Car la destruction créatrice chère à Joseph Schumpeter a éludé du modèle les catastrophes et les accidents...

«... Mais l'inimaginable nous enseigne déjà quelques austères leçons. Il nous faut réapprendre les limites, et tenter de découvrir, derrière l'indispensable sobriété, ce que signifie le mot vertu. Et puisque le pouvoir est en cause, exiger de ceux qui se veulent nos mandataires le sens du devoir qu'appelle la responsabilité à laquelle ils aspirent ${ }^{17} \gg$. 
1/ASN : Autorité de sûreté nucléaire chargée :

- de contribuer à l'élaboration de la réglementation, en donnant son avis au gouvernement sur les projets de décrets et d'arrêtés ministériels ou en prenant des décisions réglementaires à caractère technique ;

- de vérifier le respect des règles et des prescriptions auxquelles sont soumises les installations ou activités qu'elle contrôle ;

- de participer à l'information du public, y compris en cas de situation d'urgence

- IRSN : Institut de radioprotection et de sûreté nucléaire Le décret n 2016-283 du 10 mars 2016 confie à l'IRSN des missions d'expertise et de recherche dans les domaines suivants :

- la sûreté nucléaire ;

- la sûreté des transports de matières radioactives et fissiles ;

- la protection de l'homme et de l'environnement contre les rayonnements ionisants ;

- la protection et le contrôle des matières nucléaires ;

- la protection des installations nucléaires et des transports de matières radioactives et fissiles contre les actes de malveillance.

2/BEATT : Le Bureau d'enquêtes sur les accidents de transport terrestre. Créé en 2004, le BEA-TT a pour mission de réaliser, en toute indépendance, des enquêtes techniques sur les accidents ou incidents graves ou potentiellement graves de transport terrestre afin d'en établir les circonstances, d'en identifier les causes certaines ou possibles et d'émettre des recommandations de sécurité destinées à prévenir de futurs accidents similaires. Acteur de prévention contre les accidents de transport terrestre, le BEA-TT a une vocation purement technique. Ses enquêtes ne visent pas à déterminer des responsabilités. Son champ d'intervention couvre à la fois les transports ferroviaires, les transports routiers, les modes guidés urbains (métros et tramways), les remontées mécaniques et la navigation intérieure. Il dispose d'une très large capacité d'investigation, qui lui est ouverte par la loi, et a notamment accès aux dossiers d'information ou d'instruction judiciaire concernant les accidents qu'il analyse.

3/GIEC : Le Groupe d'experts intergouvernemental sur l'évolution du climat (GIEC) a été créé en 1988 par deux institutions des Nations unies : l'Organisation météorologique mondiale (OMM) et le Programme des Nations unies pour l'environnement (PNUE). Cet organisme intergouvernemental est ouvert à tous les pays membres de ces deux organisations.

Le GIEC a pour mandat d'évaluer, sans parti pris et de manière méthodique et objective, l'information scientifique, technique et socio-économique disponible en rapport avec la question du changement $\mathrm{du}$ climat. Ces informations sont sélectionnées parmi les études effectuées par des organismes pluridisciplinaires internationaux et publiées dans des revues scientifiques. Le GIEC travaille à dégager clairement les éléments qui relèvent d'un consensus de la communauté scientifique et à identifier les limites d'interprétation des résultats. La compréhension des fondements scientifiques du changement climatique provoqué par l'homme doit permettre d'en établir les conséquences et d'envisager des stratégies d'adaptation et d'atténuation. Les rapports ne doivent pas préconiser de choix de nature politique. La formule constamment rappelée à ce sujet est qu'il faut être «policy relevant, but not policy prescriptive ».

4/ARIA : la base de données ARIA (Analyse, recherche et information sur les accidents) recense les incidents ou accidents qui ont, ou auraient, pu porter atteinte à la santé ou la sécurité publique, l'agriculture, la nature et l'environnement. Pour l'essentiel, ces événements résultent de l'activité 
d'usines, ateliers, dépôts, chantiers, carrières, élevages... classés au titre de la législation relative aux installations classées. ARIA recense plus de 45000 accidents ou incidents survenus en France ou à l'étranger, près de 1300 ont été ainsi répertoriés au titre de l'année 2014. Depuis 2010, au-delà des installations industrielles et agricoles, les domaines d'accidentologie concernés ont été progressivement étendus au transport de matières dangereuses par route, fer, eau et canalisations, à la distribution et à l'utilisation du gaz, aux équipements sous pression, aux mines, carrières et stockages souterrains, ainsi qu'aux barrages et digues. 